\title{
TECNOLOGIAS APROPRIADAS AO PROCESSO DO TRABALHO DE PARTO HUMANIZADO
}

Francisca Marta de Lima Costa Souza ${ }^{1}$, Wenysson Noleto dos Santos ${ }^{1}$, Rebecca Stefany da Costa Santos ${ }^{2}$ Onadja Benicio Rodrigues ${ }^{3}$, Janmilli da Costa Dantas Santiago ${ }^{1}$, Richardson Augusto Rosendo da Silva ${ }^{1}$

Objetivo: Refletir sobre as tecnologias apropriadas utilizadas pelos enfermeiros generalistas e obstetras no processo do trabalho de parto humanizado. Metodologia: Trata-se de uma reflexão acerca das tecnologias duras, leve e leve-dura utilizadas no parto. Optou-se por pensar o processo de parto na atualidade relacionando aos conceitos de tecnologias conforme Merhy. Resultados: Foram apontados neste percurso o uso de tecnologias leve e leve-dura como ferramentas apropriadas para o parto humanizado, pois favorecem a autonomia da mulher e sua subjetividade, porém, as tecnologias duras em alguns momentos são importantes no processo parturitivo, como uma intervenção cirúrgica conforme as recomendações do Ministério da Saúde. Conclusões: A aplicabilidade das tecnologias leve e leve-dura é favorável ao parto humanizado, reduzindo assim os riscos de infecção e mortalidade materna e neonatal decorrentes de intervenções desnecessárias provenientes das tecnologias duras.

Descritores: parto humanizado, enfermeiro obstetra, obstetrícia.

\section{TECHNOLOGIES APPROPRIATE TO HUMANIZED LABOR PROCESS}

Objective: to reflect on the appropriate technologies used by generalist nurses and obstetricians in the process of humanized labor. Methodology: This is a reflection about the hard, light and light-hard technologies used in childbirth. The process of childbirth in the present time was related to the concepts of technologies according to Merhy. Results: the use of light and hard-light technologies as appropriate tools for humanized childbirth, as they favor women's autonomy and subjectivity, were pointed out in this course, however, hard technologies are sometimes important in the parturition process, such as a surgical intervention according to the recommendations of the Ministry of Health.. Conclusions: The applicability of light and hard-light technologies is favorable to humanized labor, thus reducing the risks of infection and maternal and neonatal mortality caused by unnecessary interventions from hard technologies.

Descriptors: humanizing delivery, nurse midwives, obstetrics

\section{TECNOLOGİAS APROPIADAS AL PROCEDIMIENTO DEL TRABAJO DE PARTO HUMANIZADO}

Objetivo: reflexionar sobre las tecnologías apropiadas utilizadas por los enfermeros generalistas y obstetras en el proceso del trabajo de parto humanizado. Metodología: Se trata de una reflexión acerca de las tecnologías duras, ligeras y ligeras utilizadas en el parto. Se optó por pensar el proceso de parto en la actualidad relacionando a los conceptos de tecnologías conforme a Merhy. Resultados: el uso de tecnologías de luz y luz dura como herramientas apropiadas para el parto humanizado, ya que favorecen la autonomía y la subjetividad de las mujeres, se señaló en este curso; sin embargo, las tecnologías duras a veces son importantes en el proceso de parto, como una intervención quirúrgica de acuerdo con Las recomendaciones del Ministerio de Salud. Conclusiones: la aplicabilidad de las tecnologías ligera y ligera es favorable al parto humanizado, reduciendo así los riesgos de infección y mortalidad materna y neonatal resultantes de intervenciones innecesarias provenientes de las tecnologías duras.

Descriptores: parto humanizado, enfermero obstetra, obstetricia.

1 Universidade Federal do Rio grande do Norte-UFRN.

2 Universidade Estadual do Rio Grande do Norte-UERN

Hospital e Maternidade Ana Bezerra-HUAB.

Autor correspondente: Wenysson Noleto dos Santos. E-mail: wenysson-noleto@hotmail.com 


\section{INTRODUÇÃO}

Do século XVI ao XVIII as mulheres pariam em casa com a ajuda de parteiras, comadres e curiosas que colaboravam com o nascimento do neonato de maneira não intervencionista. Assim, a gestante era protagonista no processo de parturição, o que possibilitava uma vivência mais intensa, humana, afetiva, familiar e pessoal deste processo(1).

Com o advento do capitalismo no Brasil ao raiar do século XIX, o surgimento do fórceps e a aceitação da obstetrícia como disciplina técnica, científica e dominada pelo homem, o parto passa a ser identificado como um evento perigoso e patológico para a mulher e o feto, sendo assim essencial a presença do médico no processo de parturição( ${ }^{(2)}$.

Inicialmente os médicos assistiam as parturientes em casa com a participação das parteiras. Contudo, a ideia de risco de vida para a mulher e o recém-nascido nos partos em domicílio era bastante discutida entre os obstetras, gerando, pois, a necessidade da hospitalização da gestante no momento do parto e, por conseguinte, o surgimento das maternidades.

A conquista da realização do parto hospitalizado e a criação de maternidades, tiveram resultados significativos, tais como a redução de óbitos maternos e neonatais, de modo que foram sonegados a individualidade e autonomia das mulheres. Com efeito, a imposição de rotinas pelos profissionais evidencia, portanto, a substituição do paradigma não intervencionista, deixando a mulher de ser a protagonista do processo parturitivo, restando incertos os aspectos sobre o tipo de parto, as posições de parir e a negação quanto às intervenções desnecessárias ${ }^{(3)}$.

Além dos elevados números de intervenções no parto e no nascimento, a exemplo da episiotomia e do uso do fórceps, realce-se o aumento de cesáreas, caracterizando a sobreposição da tecnologia dura em detrimento da leve e leve-dura. Ademais, destaque-se que no Brasil as operações cesarianas são o modo de nascimento mais comum, atingindo uma média de $56,7 \%$ de todos os nascimentos ocorridos no país ( $85 \%$ na rede de atenção hospitalar privada e $40 \%$ na pública). Ao revés, na França a taxa de cesárea é cerca de $20 \%{ }^{(4)}$.

Imperioso registrar que a cesariana é um procedimento essencial à saúde materna e infantil quando o parto normal não é favorável devido à distócia e/ou presença de patologias que ponham em risco a vida da mulher e do feto. No entanto, quando a cesariana é realizada sem justificativa, pode acarretar complicações como infecção ou morte materna e infantil, tornando a redução da morbimortalidade um desafio, bem como provocando a elevação desnecessária de recursos públicos ${ }^{(4-5)}$.
O excesso de intervenções obstétricas e o baixo uso de boas práticas na atenção ao parto ainda são comuns no Brasil. A taxa de recém-nascidos prematuros no Brasil $(11,5 \%)$ é quase duas vezes superior à encontrada nos países europeus. Gestações são interrompidas sem indicação, por meio de cesarianas agendadas ${ }^{(6)}$.

Frente a esta problemática torna-se imprescindivel a qualificação da atenção à gestante desde o pré-natal por meio de diálogo com a equipe de saúde, a fim de que a tomada de decisão pela via de parto considere os ganhos em saúde. As fases do pré-parto e parto são experiências marcantes na vida da mulher, e podem vir acompanhadas de sentimentos de alegria ou tristeza, e isso depende de como tenha vivido cada momento do trabalho de parto. Para que o parto seja reconhecido pelas mulheres como um ato prazeroso e fisiológico, os enfermeiros generalistas e obstetras envolvidos na assistência ao parto devem ser sensiveis para ouvir a mulher, compreender as necessidades individuais e encorajá-la a assumir sua autonomia de forma ativa neste processo.

De acordo com a Resolução COFEN № 0516/2016, no Art. 1ㅇ, normatiza a atuação e a responsabilidade do enfermeiro, enfermeiro obstetra e obstetriz na assistência às gestantes, parturientes, puérperas e recém-nascidos nos Serviços de Obstetrícia, no centro de parto normal e nas casas de parto e demais locais onde ocorra essa assistência. Aos enfermeiros obstetras ainda compete a emissão de laudos de autorização de internação hospitalar (AIH) para o procedimento de parto normal sem distócia ${ }^{(7)}$.

Face ao exposto, torna-se necessário refletir sobre as técnicas, procedimentos e orientações aplicadas à mulher no pré-parto e parto, no intuito de aproximar o nascimento da criança de um processo mais natural e saudável. Frente a este cenário, objetivou-se refletir sobre a tecnologia apropriada a ser utilizada pelos enfermeiros generalistas e obstetras no processo do trabalho de parto humanizado.

\section{METODOLOGIA}

\section{Tipo de estudo}

Trata-se de uma reflexão acerca das tecnologias duras, leve e leve-dura utilizadas no processo de trabalho de parto. Optouse por pensar o processo de parto na atualidade relacionando aos conceitos de tecnologias conforme Merhy ${ }^{(8)}$.

\section{Participantes da pesquisa}

Para construir a reflexão, realizou-se uma revisão integrativa nas bases de dados: Literatura Latino-Americana e do Caribe em Ciências da Saúde (LILACS), Bases de Dados em Enfermagem (BDENF), Scientific Eletronic Library Online 
(SciELO), e US National Library of Medicine National Institutes of Health (PubMed)," Cumulative Index to Nursing $\varepsilon$ Allied Health Literature (CINAHL) e Scopus.

Para tanto, foram realizados acessos ao portal da Coordenação de Aperfeiçoamento de Pessoal de Nivel Superior (CAPES), além de consulta a legislações relacionadas à atenção pré-natal no Brasil, manuais do Ministério da Saúde, documentos oficiais do COFEN e livros sobre a temática em estudo. Constituiu-se uma amostra de 23 artigos.

\section{Critérios de inclusão e exclusão}

Os critérios de inclusão para seleção dos artigos foram: artigos completos disponiveis gratuitamente nas bases de dados selecionadas; artigos nos idiomas português, inglês e espanhol. Como critérios de exclusão: editoriais, cartas ao editor e opinião de especialistas.

\section{Local do estudo}

A fim de embasar o estudo, realizou-se uma revisão integrativa no laboratório de informática da Universidade Federal do Rio Grande do Norte, RN, Brasil.

\section{Coleta dos dados}

Os dados foram coletados durante o período de outubro a novembro de 2017. A coleta procedeu em três etapas distintas, na primeira etapa, foram utilizados os descritores presentes nos Descritores em Ciências da Saúde (DeSC) e Medical Subject Heading (MeSH): "parto humanizado", "enfermeiro obstetra", "enfermagem", "Humanizing Delivery", "Nurse Midwives", "Nursing ", cruzados por meio do operador booleano AND, a partir dos seguintes cruzamentos 3X3: "parto humanizado" AND "enfermeiro obstetra" AND "enfermagem"; "Humanizing Delivery" AND “Nurse Midwives" AND "Nursing".

Na segunda etapa, foi realizada a pré-seleção à partir da leitura flutuante, apenas a seção de títulos e resumos. Enquanto na terceira etapa, a amostra final foi alcançada pela leitura dos manuscritos na íntegra. Por fim, foram escolhidos 32 artigos para leitura na integra nas bases elencadas e incluídos para o estudo 23 artigos. Todo o processo de coleta foi graficamente representado de acordo com o fluxograma apresentado na Figura 1. .

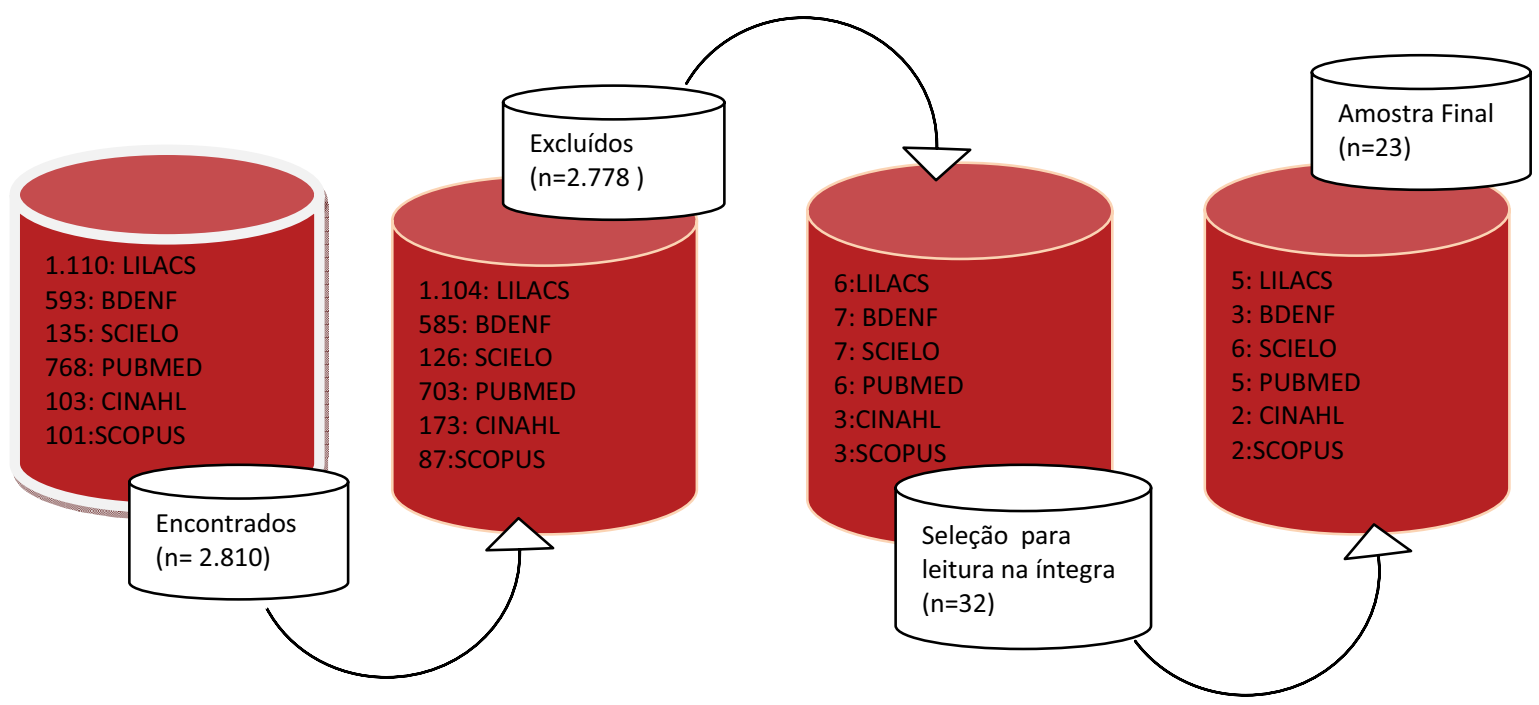

Figura 1 Fluxograma de busca dos estudos nas bases de dados. Natal/RN, 2017.

\section{Procedimentos de análise dos dados}

Para análise do material, foi realizada a leitura integral dos artigos incluídos, com a finalidade de realizar a análise direcionada pela questão condutora, quais as tecnologias apropriadas utilizadas pelos enfermeiros generalistas e obstetras no processo do trabalho de parto humanizado?

No tocante à amostra selecionada foram extraídas as seguintes variáveis: base de dados de indexação do artigo, ano de publicação, título, objetivo(s), delineamento do es- tudo, local de realização, intervenção/ tecnologia e tipo de tecnologia.

\section{Procedimentos éticos}

Por se tratar de uma a reflexão por meio uma revisão integrativa cuja fonte de dados é de domínio público e de livre acesso, o presente estudo não necessitou de apreciação por parte do Comitê de Ética em Pesquisas com Seres Humanos. 


\section{RESULTADOS}

Por meio da análise dos 23 artigos selecionados, verificou-se que a maioria ( 8 - 34\%) dos estudos foi encontrada na base de dados Scielo. Observou-se que prevaleceram as publicações ocorridas a partir do ano 2015 (7 - 30\%), realizados pelos Estados Unidos da América (EUA) (2 - 8\%), cujo idioma era o inglês ( $9-39,1 \%)$.

Verificou-se que alguns artigos traziam mais de um tipo de tecnologia para a promoção do parto humanizado, assim foram identificados 32 tipos de tecnologia para o cuidado, as quais foram classificadas como leve-dura (15 $46 \%$ ) e leve (13 - 40\%). Além disso, o acolhimento (9-39\%), o estímulo à deambulação (6 - 26\%), a ambientação (5 $21,7 \%)$, a bola de bobath $(5-21,7 \%)$ e as massagens relaxantes (5 - 21,7\%), aromaterapia, banho de imersão, foram as tecnologias mais mencionadas.

\section{DISCUSSÃO}

As tecnologias envolvidas no processo de trabalho em saúde são conceituadas em três tipos de classificação: leve, que caracteriza as tecnologias de relação interpessoal entre sujeitos (profissionais de saúde e usuário), autonomia e acolhimento do usuário; leve-dura é um saber bem estruturado que opera no processo de trabalho em saúde; e duras, que são os equipamentos tipo instrumentais, máquinas utilizados para os procedimentos executados pelos profissionais $^{(8)}$.

No âmbito da assistência à parturiente no segundo período clínico do trabalho de parto, o uso das tecnologias duras tem se destacado por meio de intervenções muitas vezes desnecessárias, como o fórceps, episiotomia e cesarianas $^{(9)}$, que podem acarretar traumas e danos à saúde da mulher e da criança, os quais podem ser evitados se a fisiologia da gestante for respeitada.

Para mudar a realidade deste cenário, o enfermeiro pode contribuir por meio da inter-relação, da comunicação, do acolhimento, do estabelecimento de vínculos, da promoção de autonomia e empoderamento da gestante; influxos da tecnologia leve ${ }^{(10)}$. Além do uso da tecnologia leve como ferramenta de trabalho, o enfermeiro precisa também amparar-se nas tecnologias leve-duras para que as orientações repassadas às parturientes sejam estruturadas, fundamentalmente na literatura e baseadas no mais alto nível de evidências, de modo a levar a efeito as melhores técnicas relacionadas ao tipo de parto escolhido pela mulher(11-14).

Para a mulher, a gravidez e o nascimento em particular, são eventos únicos repletos de fortes sentimentos e emoções. A experiência vivida por ela nesses momentos ficará firmemente marcada em sua memória, e por isso, todos os envolvidos na sua assistência, devem the proporcionar um ambiente de carinho e humanismo ${ }^{(15)}$

Ademais, esta deve ser tratada com carinho, e deve-se respeitar o seu tempo, proporcionando o alívio da dor através de exercícios, massagens, banhos, deambulação e até mesmo adoção de posições durante o trabalho de parto ${ }^{(2-4)}$.

Segundo o modelo de atenção humanizada à parturiente, os profissionais devem permanecer presentes durante todo o trabalho de parto, oferecendo às mulheres o apoio psicológico e emocional na parturição, além de técnicas de relaxamento e massagens, música ou quaisquer outras práticas alternativas que tragam alívio e conforto à gestante(3,16).

Além disso, o movimento de humanização do parto, luta pela diminuição das intervenções desnecessárias e pela promoção de cuidado ao processo de gravidez/parto/nascimento/amamentação, que são entendidos como processo singular, natural e fisiológico e que requer o fortalecimento do papel da mulher como protagonista nesse processo(17).

O uso das boas práticas na atenção ao parto preconizado pela organização Mundial da Saúde, desde 1996, destaca como prática eficaz o acolhimento, as orientações e a liberdade da mulher quanto à posição e movimento durante o trabalho de parto ${ }^{(10,18)}$. Assim, os saberes e habilidades dos enfermeiros no processo de trabalho de parto devem estar centrados no cuidado à parturiente, com vista a aplicar técnicas de forma humanizada, inserindo um familiar da gestante neste processo, de modo a respeitar-se a fisiologia do pré-parto e parto. Desta feita, sendo o parto é um evento natural, que não necessita de controle, perseguese, com esta prática, afastar o parto tecnocrático, centrado no profissional médico ${ }^{(19)}$

Autores esclarecem que para que ocorra uma mudança na cultura hospitalocêntrica e médica, deve haver sobretudo, uma transformação na postura das equipes e profissionais para que a fisiologia do parto seja respeitada, destacam ainda que a gestante deve ser informada sobre todos os procedimentos a que será submetida, podendo inclusive apontar aqueles que não quer ${ }^{(1-3)}$. Desta forma, a mulher deve ter acesso a todas as informações baseadas em evidências e serem incluídas no processo de tomada de decisões, sendo essencial que os profissionais estabeleçam um vínculo de confiança, sempre tornando-se ciente dos desejos e expectativas da parturiente ${ }^{(6,20)}$.

Estudo realizado em uma maternidade pública apontou que as enfermeiras obstétricas perceberam que o relaxamento da mulher na água quente durante o banho de aspersão interfere na progressão do trabalho de parto: o banho auxilia a acelerá-lo já que, a partir do relaxamento, há um rápido acréscimo da dilatação do colo uterino, favorecendo uma descida mais rápida e adequada do bebê $\hat{e}^{(5)}$. 
Assim, o banho de imersão é um método muito utilizado pelas participantes na assistência ao trabalho de parto e parto, favorecendo a atenção obstétrica e evitando práticas intervencionistas, desse modo, promovendo uma resposta satisfatória quanto ao relaxamento da mulher e o declínio das catecolaminas no organismo(21).

Já o banho de imersão é classificado como uma tecnologia de alívio da dor durante o trabalho de parto, por proporcionar à mulher a retomada de sua autonomia no processo de parturição, pois pode mobilizar seus próprios recursos na busca de seu bem estar durante esse momento. Além disso, essa prática contribui para que as mulheres vivenciem o processo do parto de maneira mais harmoniosa e relaxante(22). Considerando que os projetos de investimentos das maternidades estão somente nos discursos políticos e institucionais, faz-se necessário investimento para a construção e adaptação das maternidades, seguindo os modelos para garantir a humanização da assistência(9)

A aromaterapia é um tratamento complementar não medicalizado, que usa os sentidos do toque e do cheiro. Cada vez mais utilizados, os óleos essenciais com aroma delicado, levemente doce, por vezes aveludado, cítrico, amadeirado, como a lavanda, o eucalipto, o jasmim, a rosa e a laranja, estão trazendo efeitos significativos na percepção da dor, na ansiedade das parturientes e, consequentemente, na duração das fases do processo parturitivo, quando a escolha bem aplicada inspira um toque de sensibilidade e sabores ${ }^{(23)}$. Ao ser inalado, o óleo impulsiona os receptores sensitivos através do cérebro, envolvendo partes neurológicas específicas e substâncias que se harmonizam de maneira inebriante, resultando em mudanças físicas e psicológicas, capazes de obter resultados efetivos na redução da dor e da ansiedade ${ }^{(10)}$

Estudo revela que profissionais atenciosos, acolhedores e comunicativos podem contribuir na qualidade dos cuidados, no processo de trabalho de parto, conquanto, os profissionais precisam ser preparados para melhorar essas qualidades, tendo em vista que o cuidado orientado pelo conceito de humanização é aquele que respeita a dignidade, os direitos e autonomia da mulher. Logo, o parto deve ser compreendido em sua singularidade, mas não engessado em regras e padrões, a serem obedecidas e não por isso sem segurança ${ }^{(11)}$.

Dessa forma, entende-se que o parto humanizado é aquele que tem sua fisiologia respeitada, com menos intervenções desnecessárias pelos prestadores de assistência, com estímulo ao protagonismo da mulher, a presença de acompanhante de sua livre escolha, bem como por meio de práticas comprovadas cientificamente como benéficas para mãe e o recém-nascido, tais como o aleitamento na primeira hora de vida, o contato pele a pele precoce, o clampeamento de cordão após cessar a pulsação, entre outros $^{(3)}$

O enfermeiro deve dar suporte afetivo, psicológico, físico-técnico e tecnológico à parturiente, intervindo apenas quando realmente necessário. Trata-se, portanto, de processo que deve ocorrer em sintonia com os movimentos subjetivos da atualidade. que prezam pelo respeito à temporalidade singular de cada parto e, principalmente, acreditam na potência feminina para parir, seja em ambiente domiciliar ou hospitalar.

Assim, é possivel potencializar o saber para o desenvolvimento de práticas de cuidados que não sejam invasivas à fisiologia do corpo, empoderando a mulher a viver os momentos de prazer que o parto lhe proporciona ${ }^{(12)}$.Visualizar a mulher com sujeito ativo do parto facilita a aplicabilidade de técnicas não invasivas que contribuam com o parto. As parturientes precisam de ambiente acolhedor, privacidade e orientações, e, para atender essas necessidades essenciais ao processo parturitivo, as maternidades, desde 2009 , vêm substituindo o modelo de sala de parto pela implantação de suítes PPP (pré-parto, parto e pós-parto), ambiente este de práticas inovadoras e recomendáveis na assistência ao parto e nascimento.

Outra medida que vem contribuindo com o processo de trabalho de parto humanizado é a participação do acompanhante no parto, regularizado pela lei de n. 1l. 108, de 7 de abril de 2005. Aliás, é inconteste que o acompanhante, escolhido pela parturiente, tem contribuido no partejamento e no suporte emocional à mulher.

Além das suítes PPPs e da participação do acompanhante no pré-parto e parto, os enfermeiros obstetras, por estarem sempre presentes no acompanhamento do trabalho de parto, reafirmam a importância do estímulo à aplicação dos métodos não famacológicos para o alívio da dor, no primeiro período do trabalho de parto, como: banhos quentes, massagens, deambulação, uso da bola de bobath, balanço pélvico, as posições que favorecem a descida do feto, tipo vertical ${ }^{(23)}$.

Impende destacar ainda ser perceptível que o uso das tecnologias leve e leve-dura tem contribuido no fortalecimento a autonomia da mulher, incentivando-a a decidir quanto à posição de parir e à ocorrência do hands off. Estudos realizados quanto ao parto evidenciaram a eficácia das medidas não farmacológicas aplicadas no pré-parto (13-14,20-21)

É possivel avançar nesta perspectiva utilizando as tecnologias apropriadas, e, como meio de contribuir na redução dos partos cesarianas e aumentar os partos humanizados, ${ }^{(22)}$ é indispesável contar com a participação dos enfermeiros que atuam na atenção primária à saúde, os 
quais devem fortalecer as orientações à mulher no prénatal quanto ao parto, a elaboração do plano de parto e o incentivo à participação de uma "doula" no partejar(23).

\section{Limitações do estudo}

Quanto às limitações do estudo, ressalta-se a ausência de busca em outras bases de dados, tais como: Web of Science, Science Direct, Cochrane.

\section{Contribuições do estudo para a prática}

O estudo apresenta contribuições para a área da enfermagem e saúde ao refletir sobre o uso de tecnologias leves e leve-duras - com destaque para a potencialização do saber voltado à elaboração e desenvolvimento de práticas de cuidados relacionados aos processos de gestar e parir que não sejam invasivas à fisiologia do corpo, da mente e privacidade do ser feminino. Assim, o caráter não invasivo das tecnologias de cuidado da enfermagem obstétrica reside em acreditarmos que, quando o sujeito estabelece um vínculo de confiança com o profissional, ambos compartilham as decisões no planejamento dos seus cuidados.

\section{CONCLUSÕES}

O estudo permitiu identificar como tecnologias apropriadas utilizadas pelos enfermeiros generalistas e obstetras no processo do trabalho de parto humanizado: acolhimento, o estímulo à deambulação, a ambientação, a bola de bobath e as massagens relaxantes.

A incidência das tecnologias leve e leve-dura é favorável ao parto humanizado, na medida em que reduz os riscos de infecção e mortalidade materna e neonatal. Por seu turno, as tecnologias duras, como o fórceps e episiotomia, caracterizam risco a saúde materna-infantil, devendo, portanto, ser evitadas.
Ademais, as tecnologias para melhorar os desfechos maternos relacionados às boas práticas de assistência ao parto humanizado indicam a necessidade de respeito, relações efetivas no trabalho, resolutividade no atendimento, acesso às informações entre os membros da equipe e entre estes e os usuários.

É importante a implementação das práticas baseadas em evidência na assistência ao parto humanizado para contribuir na redução dos altos índices de cesárea que atualmente o Brasil vem registrando. É possível empoderar a parturiente, conferindo-lhe autonomia para decidir o tipo de parto desejado.

Espera-se, neste cenário a redução das intervenções desnecessárias, a violência obstétrica, e o fortalecimento dos partos hands off. Por fim, conclui-se que no processo de trabalho de parto o uso da tecnologia dura, como a cesariana, é necessária em alguns desfechos desfavoráveis do trabalho de parto. Todavia, as ferramentas leve e levedura são primordiais para o parto humanizado, tendo em vista, sobremodo que os enfermeiros obstetras são profissionais comprometidos e qualificados capazes de resgatar o parto como evento fisiológico, que respeita autonomia da mulher.

\section{Contribuição dos autores}

Francisca Marta de Lima Costa Souza, Wenysson Noleto dos Santos, Rebecca Stefany da Costa Santos, Onadja Benicio Rodrigues, Janmilli da Costa Dantas Santiago, concepção e desenho, análise e interpretação dos dados, redação do artigo, revisão crítica. Richardson Rosendo Augusto da Silva: concepção e desenho, análise e interpretação dos dados, redação do artigo, revisão crítica, revisão final. 


\section{REFERÊNCIAS}

1. Menezes PFA, Portella SDC, Bispo TCF. A Situação do parto domiciliar no Brasil. Revista Enfermagem Contemporânea [internet]. 2012 [Cited 2019 jan 12]; 1(1):3-43. Available from: https://www5. bahiana.edu.br/index.php/enfermagem/article/view/38.

2.Velho MB, Oliveira ME, Santos EKA. Reflexões sobre a assistência de enfermagem prestada à parturiente. Rev Bras Enferm [internet]. 2010 [Cited 2019 jan 12]; 63(4): 652-9. Available from: https://www. redalyc.org/pdf/2670/267019592023.pdf

3. Ministério da Saúde (BR). Cadernos Humaniza SUS: Humanização do parto e nascimento. [Internet]. Brasilia: Ministério da Saúde; 2014 [Cited 2019 jan 12]. Available from: http://www.redehumanizasus.net/sites/default/files/caderno_humanizasus_v4_humanizacao_parto.pdf.

4. Ministério da Saúde (BR). Secretaria de Ciência, Tecnologia e Insumos Estratégicos Diretrizes de Atenção à gestante: a operação cesariana. [Internet]. Brasilia: Ministério da Saúde; 2016 [Cited 2019 jan 12];179.Available:http://conitec.gov.br/images/Consultas/ Relatorios/2015/Relatorio_PCDTCesariana_CP.pdf.

5. Victora CG, Aquino EM, Leal MC, Monteiro CA, Barros FC, Szwarcwald CL. Maternal and child health in Brazil: progress and challenges. Lancet [Internet]. 2011. [Cited 2019 jan 15]; 377(9780): 1863-76. Available from: https://www.ncbi.nlm.nih.gov/pubmed/21561656.

6. Leal MC, et al. Prevalence and risk factors related to preterm birth in Brazil. Reproductive Health [Internet]. 2016 [Cited 2019 jan 15]; 13(3):127. Available from: https://www.ncbi.nlm.nih.gov/pmc/articles/PMC5073982/

7.Conselho Federal de Enfermagem. Resolução COFEN № 0516/2016. Portal COFEN [Cited $2016 \mathrm{dez}$ 10]. Available from: http://www.cofen. gov.br/resolucao-cofen-no-05162016_41989.html.

8. Merhy EE. A cartografia do trabalho vivo. Hucitec [Internet]. 2017 [Cited 2019 jan 15]; Rio de Janeiro, 24(8):1953-7. Available from: http://www.scielo.br/pdf/csp/v24n8/23.pdf.

9. Amorin MMR, Porto AMF, Souza ASR. Assistência ao segundo e terceiro periodos do trabalho de parto baseada em evidências. Rev Fem [Internet]. 2010 [Cited $2016 \mathrm{dez}$ 15]; 11(38): 583-91. Available from: http://files.bvs.br/upload/S/0100-7254/2010/v38nl1/a583-591.pdf.

10. Ministério da Saúde (BR). Manual de Acolhimento e Classificação de Risco em Obstetricia. Rede Cegonha/DAPES/SAS-DF [Internet]. Brasilia: Ministério da Saúde; 2014[Cited 2016 dez 15]. Available from: http://bvsms.saude.gov.br/bvs/publicacoes/manual_acolhimento_classificacao_risco_obstetricia.pdf.

11. Baldisserotto ML, Theme Filha MM, Gama SGN. Good practices according to WHO's recommendation for normal labor and birth and women's assessment of the care received: the "birth in Brazil" national research study, 2011/2012. Reproductive Health [Internet]. 2016 [Cited $2016 \mathrm{dez} 16$ ]; 13(3):124. Available from: https://www.ncbi.nlm. nih.gov/pmc/articles/PMC5073993/.

12. Nascimento NM, Progianti JM, Novoa RI, Oliveira TR, Vargens OMC. Tecnologias não invasivas de cuidado no parto realizadas por enfermeiras: a percepção de mulheres. Esc Anna Nery [Internet]. 2010 [Cited $2016 \mathrm{dez}$ 16]; 14 (3):456-61. Available from: http://www. scielo.br/scielo.php?pid=S1414-814520100003000048script=sci_ abstractetlng=pt.

13. Côrtes CT, Santos RCS, Caroci AS, Oliveira SG, Oliveira SMJV, Riesco MLG. Metodologia de implementação de práticas baseadas em evidências cientificas na assistência ao parto normal: estudo piloto. Rev Esc Enferm USP [Internet]. 2015 [Cited 2016 dez 16]; 49(5):716-725. Available from: http://www.scielo.br/pdf/reeusp/ v49n5/pt_0080-6234-reeusp-49-05-0716.pdf.

14. Pereira ALF, Lima TRL, Schroeter MS, Gouveia MSF, Nascimento SD. Resultados maternos e neonatais da assistência em casa de parto no municipio do Rio de Janeiro. Esc Anna Nery [Internet]. 2013 [Cited $2016 \mathrm{dez}$ 16];17(1):17-23. Available from: http://www.scielo.br/ scielo.php?script=sci_arttextepid=\$1414-81452013000100003.

15. Maurer CEC, Atherino SEK, Andréia NA, Marisa M. O processo de parir assistido pela enfermeira obstétrica no contexto hospitalar: significados para as parturientes. Esc Anna Nery [Internet]. 2012 [cited 2019 July 22]; 16(1):34-40. Available from: http://www.scielo. br/scielo.php?script=sci_arttextSpid=\$1414-81452012000100005slng=en.

16. Pinheiro BC, Bittar CML. Expectativas, percepções e experiências sobre o parto normal: relato de um grupo de mulheres. Fractal: Revista de Psicologia [Internet]. 2013 [cited 2019 July 22]; 25(3):585-602. Available from: http://www.scielo.br/pdf/fractal/ v25n3/allv25n3.pdf

17. Prata JA, Progianti JM. A influência da prática das enfermeiras obstétricas na construção de uma nova demanda social. Revista Enfermagem UERJ[Internet]. 2013 [cited 2019 July 22]; 21(1):23-8. Available from: https://www.e-publicacoes.uerj.br/index.php/enfermagemuerj/article/view/6341

18. Barbieri M, Henrique AJ, Chors FM, Maia NL, Gabrielloni MC. Banho quente de aspersão, exercicios perineais com bola suiça e dor no trabalho de parto. Acta paul enferm [Internet]. 2013 [cited 2019 July 22];26(5):478-84. Available from: http://www.scielo.br/scielo. php?script=sci_arttextopid=s0103-210020130005000128lng=en. http://dx.doi.org/10.1590/S0103-21002013000500012.

19. Kaviani M, Machbool S, Azima S, Tabael MH. Comparison of the effect of aromatherapy with Jasminum officinale and Salvia officinale on pain severity and labor outcome in nulliparous women. Iran J Nurs Midwifery Res [Internet]. 2014 [cited 2019 July 21]; 19(6). Available from: https://www.ncbi.nlm. nih.gov/pmc/articles/ PMC4280734/.

20. Motta SAMF, Feitosa DS, Bezerra STF, Dodt RCM, Moura DJM. Implementação da humanização da assistência ao parto natural. Rev enferm UFPE on line[Internet]. 2016 [cited 2019 July 21];10(2):593-9. Available from: https://pdfs.semanticscholar.org/49d4/52c92e0fe 4 1f77c85de7250c4b0e71609603.pdf

21. Enderle CF, Kerber NPC, Susin LRO, MendozaSassi RA. Avaliação da atenção ao parto por adolescentes em um hospital universitário. Rev Bras Saude Mater Infant [Internet]. 2012 [cited 2015 Out 10];12(4):383-94. Available from: http://www.scielo.br/pdf/rbsmi/ v12n4/a05v12 n4.pdf

22. Guerra CS, Brito LPB, Moraes MN, Cordeiro RC, Araujo VS, Dias MD. The importance of care provided to women by traditional midwives during household childbirth. J Nurs UFPE on line[Internet]. 2013 [cited 2013 Oct 12];7(8):5214-9. Available from: www.revista.ufpe.br/ revistaenfermagem/index. php/revista/article/.../6937

23. Souza VB, Silva JS, Barros MC, Freitas PSP. Tecnologias leves na saúde como pontencializadores para qualidade da assistência às gestantes. Rev enferm UFPE on line[Internet]. 2014 [cited 2013 Oct 12];8(5):1388-93. Available from: file:/I/C:/Users/Win7/ Downloads/9824-18512-1-PB.pdf 